2018-05-01

\title{
Raman Spectral Analysis for Rapid Screening of Dengue Infection
}

Tahir Mahmood

Department of Chemistry, University of Agriculture, Faisalabad, Pakistan.

Haq Nawaz

Department of Chemistry, University of Agriculture, Faisalabad, Pakistan.

A. Ditta

Department of Chemistry, University of Agriculture, Faisalabad, Pakistan.

See next page for additional authors

Follow this and additional works at: https://arrow.tudublin.ie/biomart

Part of the Biochemistry Commons, Biological and Chemical Physics Commons, and the Other Analytical, Diagnostic and Therapeutic Techniques and Equipment Commons

\section{Recommended Citation}

Mahmood, T., Nawaz, H. \& Ditta, A. (2018). Raman Spectral Analysis for Rapid Screening of Dengue Infection. Spectrochimica Acta Part A: Molecular and Biomolecular Spectroscop, Jul. 5, no. 200, pg. 136-142. doi: 10.1016/j.saa.2018.04.018

This Article is brought to you for free and open access by the Biomedical and Environmental Sensing at ARROW@TU Dublin. It has been accepted for inclusion in Articles by an authorized administrator of ARROW@TU Dublin. For more information, please contact arrow.admin@tudublin.ie, aisling.coyne@tudublin.ie, gerard.connolly@tudublin.ie.

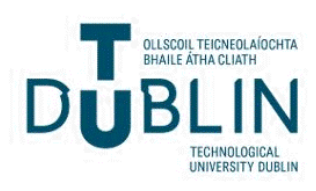




\section{Authors}

Tahir Mahmood, Haq Nawaz, A. Ditta, M.I. Majeed, M.A. Hanif, N. Rashid, H.N. Bhatti, H.F. Nargis, M. Saleem, Franck Bonnier, and Hugh Byrne 
Title:

Raman spectral analysis for rapid screening of dengue infection

\section{Authors:}

T. Mahmood ${ }^{\mathrm{a}}$ H. Nawaz ${ }^{\mathrm{a}}$, A. Ditta ${ }^{\mathrm{a}}$, M. I. Majeed ${ }^{\mathrm{a}}$, M. A. Hanif ${ }^{\mathrm{a}}$, N. Rashid ${ }^{\mathrm{b}}$, H. N. Bhatti ${ }^{\mathrm{a}}$, H. F. Nargis ${ }^{\mathrm{a}}$, M. Saleem ${ }^{\mathrm{c}}$, F. Bonnier ${ }^{\mathrm{d}}$ and H.J. Byrne ${ }^{\mathrm{e}}$

a Department of Chemistry, University of Agriculture, Faisalabad, Pakistan.

${ }^{\mathrm{b}}$ University of Central Punjab, Faisalabad campus, Faisalabad, Pakistan.

${ }^{\mathrm{c}}$ National Institute of Lasers and Optronics (NILOP), Islamabad, Pakistan.

${ }^{\text {d}}$ EA 6295 Nano-médicaments and nano-sondes, Université François-Rabelais de Tours, Tours, France.

${ }^{\text {e }}$ FOCAS Research Institute, Dublin Institute of Technology, Kevin Street, Dublin 8, Ireland.

*Corresponding Author: Dr Haq Nawaz

E-mail: haqchemist@yahoo.com 


\begin{abstract}
Infection with the dengue virus is currently clinically detected according to different biomarkers in human blood plasma, commonly measured by enzyme linked immunosorbent assays, including non-structural proteins (Ns1), immunoglobulin $\mathrm{M}(\operatorname{IgM})$ and immunoglobulin $\mathrm{G}$ ( $\operatorname{IgG})$. However, there is little or no mutual correlation between the biomarkers, as demonstrated in this study by a comparison of their levels in samples from 17 patients. As an alternative, the label free, rapid screening technique, Raman spectroscopy has been used for the characterisation/diagnosis of healthy and dengue infected human blood plasma samples. In dengue positive samples, changes in specific Raman spectral bands associated with lipidic and amino acid/protein content are observed and assigned based on literature and these features can be considered as markers associated with dengue development. Based on the spectroscopic analysis of the current, albeit limited, cohort of samples, Principal Components Analysis (PCA) coupled Factorial Discriminant Analysis, yielded values of $97.95 \%$ sensitivity and $95.40 \%$ specificity for identification of dengue infection. Furthermore, in a comparison of the normal samples to the patient samples which scored low for only one of the biomarker tests, but high or medium for either or both of the other two, PCA-FDA demonstrated a sensitivity of $97.38 \%$ and specificity of $86.18 \%$, thus providing an unambiguous screening technology.
\end{abstract}

Keywords: dengue infection; blood plasma, blood plasma biomarkers, Raman spectroscopy; Principal components analysis; Factorial Discriminant analysis; 


\section{Introduction}

Dengue disease has become a serious health concern, affecting millions of people worldwide every year. According to the WHO [1], about 3.97 billion people live in dengue pandemic tropical areas, and annually 400 million are infected, 96 million present clinically and 20,000 die of the disease. In Pakistan, 539 deaths having been reported in the last 12 years, 55,946 persons were found to be infected and $60 \%$ of cases are thought not to be reported. Dengue is a mosquito bite infection transmitted by one of the four dengue virus serotypes [2]. It is spreading more quickly in urban and semi urban areas and has become a serious health problem [3]. In some cases, the disease develops into severe DSS (dengue shock syndrome) or DHF (dengue hemorrhagic fever), resulting in bleeding leading to thrombocytopenia, loss of blood plasma, and severely low blood pressure. Children ( $\leq 15$ years) are more severely infected than adults by dengue in Asia [3-6].

Dengue viruses contain a single strand of RNA of flavivirus [7] and produce new viral strands, translated and duplicated after infection. The non-structural proteins (Ns1) in the flavivirus regulate the duplication procedure of viral RNA [8]. The Ns1 proteins circulate in the blood plasma of dengue infected patients after the onset of symptoms of dengue fever for the first few days [9] and hence can be considered a viable biomarker for the diagnosis of disease by PCR (polymerase chain reaction) and ELISA (enzyme linked immunosorbent assay) in the early stage of infection [10-11].

Acute dengue infection can be confirmed by the presence of $\operatorname{IgM}$ (immunoglobulin M), while secondary dengue virus infection is indicated by the presence of $\operatorname{IgG}$ (immunoglobulin $\mathrm{G}$ ) and both are also monitored by ELISA. Notably, the presence of IgM in a sample can indicate early stage infection, whereas the presence of elevated IgG levels, with normal IgM values, leads to the conclusion that dengue infection has occurred in the past [12].

In the early stages, a comprehensive analysis of dengue infection or biochemical changes produced by the dengue virus is of significant value for the diagnosis of dengue and its prevention. Although the treatment of dengue is only symptomatic, because there is no specific cure available for it [13], efficient and accurate diagnosis of the disease is important for clinical care which includes early detection of severe cases, case confirmation as well as differential diagnosis with other infectious diseases. Moreover, early diagnosis may also be required for outbreak control, pathogenesis, 
academic research, vaccine development, and clinical trials [14]. There are many diagnostic methods already used in clinical practice, based on the detection of RNA, antibodies in serological tests and virus isolation in cell culture etc. [15-16]. The most common clinically employed diagnostic methods for dengue detection are ELISA and PCR, which has higher specificity and sensitivity, but these modalities are usually complex, lengthy and costly. Moreover, for the detection of Ns1, IgG and IgM, separate methods/tests are used. It is a most challenging task to diagnose infection with the dengue virus in the initial stages of dengue attack, essential to prevent a pandemic, and for recovery of the patient. Where rapid detection is required, compromise has to be made on specificity and sensitivity.

Raman spectroscopy has been proven in recent years to be a highly sensitive and specific technique for the definition of biomedical changes produced in the body and diagnosis of different diseases [17-18]. It has been extensively employed over the past two decades for the characterisation of biological cells and tissue [19] and the influence of external agents on the cell [20-25]. Moreover, Raman spectroscopy has the capability to provide high content information, and even small changes in any of the biological macromolecules, including lipids, proteins and DNA, can be characterised simultaneously [26-28]. The Raman spectral changes which are identified could be helpful as diagnostic markers for the disease.

Recently, viral infections such as human papilloma (HPV), Hepatitis C (HCV), dengue virus and many other diseases have been successfully monitored by Raman spectroscopy [29-32]. Khan et al. demonstrated the potential of Raman spectroscopy, coupled with support vector machine analysis, to diagnose dengue infection in human serum, based on positive IgM status, with a high degree of accuracy (85\%) and precision (90\%), and corresponding sensitivity and specificity of $73 \%$ and $93 \%$, respectively [33]. Bilal et al. performed a similar analysis, based on Partial Least Squares Regression analysis of Raman spectroscopic data of IgM positive and normal human serum samples, and using the receiver operating characteristic curve (ROC) approach, reported an accuracy of $96.67 \%$, a sensitivity of $90 \%$ and a specificity of $100 \%$ [34]. However, in both studies, spectroscopic data was correlated with only one of the three common clinical biomarkers.

In the present study, correlations between the levels $\mathrm{Ns} 1, \mathrm{IgG}$ and $\mathrm{IgM}$ positive blood plasma samples as measured using conventional ELISA kits, are compared and it is observed that there are few or no correlations. Raman spectroscopy, coupled with Principal Components Analysis 
(PCA) is explored for differentiation between normal/healthy and Ns1, IgG and IgM positive blood plasma samples, which could pave the path for a more comprehensive monitoring of the stage of the infection by establishing specific Raman spectral features associated with these three markers of the disease. A combination of PCA and Factorial Discriminant Analysis (PCA-FDA) is employed to quantify the diagnostic potential of the technique.

\section{Materials and methods}

\section{Sample preparation}

Blood plasma samples of 17 control (healthy volunteers) and 17 patients who presented with symptoms of dengue infection, were collected from Allied Hospital Faisalabad, Pakistan. All the blood plasma samples were handled according to the US NIH (National Institute of Health) recommendations. Quantitative serology of the 17 patient samples was performed to measure the levels of NS1 protein, IgG and IgM by using ELISA kits for dengue (Vircell Cat No. G1018, M1018). The outputs of the test kits are expressed in terms of optical density, and for this study, the cut off values for Ns1, $\operatorname{IgM}$ and $\operatorname{IgG}$ were $0.389,0.378$ and 0.384 respectively, as prescribed by the manual for the respective kits. In this regard, it is important to mention that these cut off values for the Ns1, IgM and IgG can be found only for the dengue infected patients and not for the healthy/control samples, as all the three biomarkers are associated specifically with the infection of the virus. The different values of Ns1, IgM and IgG, are shown in Table 1, along with their categorisation according to Normal, Medium and High levels of biomarker.

\section{Raman spectral acquisition}

To minimise any effects of heating due to residual absorbance in plasma at the laser wavelength of $785 \mathrm{~nm}$, a $20 \mu \mathrm{l}$ drop of each plasma sample was placed on an aluminium slide at room temperature and Raman spectra were acquired before the plasma sample had dried. The process of placing the $20 \mu \mathrm{l}$ drop on the aluminium slide was repeated 3 times by employing a clean aluminium slide each time for acquiring 20-25 spectra in total from each sample.

Raman spectral acquisition from all of the 17 healthy control and 17dengue infected blood plasma samples was performed using a Raman spectrometer (Peak Seeker Pro-785; Agiltron, USA). The 
Peak Seeker Pro-785 utilizes a 785nm diode laser as the excitation source, delivering a laser power of $\sim 60 \mathrm{~mW}$ at the sample. The laser was delivered to the sample through a $10 \times$ objective. The acquisition of the Raman spectra for all the samples was executed from 600 to $1800 \mathrm{~cm}^{-1}$ and 20 25 Raman spectra per sample were acquired with an acquisition time of 30 second.

\begin{tabular}{|lllllll|}
$\begin{array}{l}\text { Dengue } \\
\text { Sample }\end{array}$ & Ns1 values & $\begin{array}{l}\text { Ns1 Cut- } \\
\text { off(0.389) }\end{array}$ & IgM values & $\begin{array}{l}\text { IgM Cut- } \\
\text { off(0.378) }\end{array}$ & IgG values & $\begin{array}{l}\text { IgG Cut- } \\
\text { off(0.384) }\end{array}$ \\
\hline D1 & 2.993 & High & 0.35 & Low & 0.136 & Low \\
\hline D2 & 3.602 & High & 0.251 & Low & 2.013 & High \\
\hline D3 & 2.727 & High & 0.143 & Low & 1.173 & High \\
\hline D4 & 2.634 & Medium & 0.155 & Low & 0.161 & Low \\
\hline D5 & 0.205 & Low & 0.88 & Medium & 1.274 & High \\
\hline D6 & 0.1 & Low & 1.252 & High & 1.997 & High \\
\hline D7 & 2.751 & Medium & 1.827 & High & 0.83 & Low \\
\hline D8 & 2.996 & High & 0.144 & Low & 0.136 & Low \\
\hline D9 & 2.786 & High & 0.727 & Medium & 0.823 & Medium \\
\hline D10 & 2.751 & High & 0.247 & Low & 1.212 & High \\
\hline D11 & 0.967 & Medium & 1.038 & High & 0.167 & Low \\
\hline D12 & 0.016 & Low & 0.046 & Low & 0.633 & Medium \\
\hline D13 & 0.125 & Low & 0.18 & Low & 0.145 & Low \\
\hline D14 & 2.93 & High & 0.173 & Low & 0.191 & Low \\
\hline D15 & 2.625 & Medium & 0.486 & Medium & 0.578 & Medium \\
\hline D16 & 1.19 & Medium & 0.117 & High & 0.118 & Low \\
\hline D17 & 2.786 & High & 0.25 & Low & 0.365 & Low \\
\hline
\end{tabular}

Table 1: Details of dengue samples and their different serologically determined values of the biomarkers Ns1, IgM and IgG. Numbers in (brackets) define the clinical cut off between normal and infected. For the purpose of this study, the latter categories are further differentiated according to "Medium" and "High" biomarker levels.

\section{Data pre-processing}

All data processing of the Raman spectra was performed using MatLab 7.8 and established protocols [26]. Data pre-processing included baseline correction, smoothing, vector normalisation, and substrate removal. All spectra, including substrate backgrounds, were vector normalised and smoothed using a Savitzky-Golay smoothing method (order 3, 17 point window). A rubber band 
correction for baseline removal for all the spectra was carried out and the substrate spectra were subtracted from each spectrum.

\section{Data analysis}

The Raman spectral features were analysed by comparing the mean Raman spectra of dengue infected blood plasma samples with healthy ones. The assignments of the Raman spectral features used in interpretation of the results were taken from the literature [25, 35-38] and are described in

Table 2. In order to further elucidate the biochemical basis of the development of dengue as compared with the healthy/control samples, PCA was performed. PCA is a mathematical procedure involving the transformation of possibly correlated variables into a smaller number of uncorrelated variables, known as principal components (PC), basically to reduce the dimensionality of the data whilst maintaining their variability. The first principal component accounts for the dominant source of variability in the data, and each succeeding principal component accounts for the next highest source of the remaining variability. The loadings of the PC can be understood as the orthogonal dimensions of biochemical differences which facilitate separation of different groups of spectra of Raman data along their variability as each spectrum scores along these dimensions.

PCA was employed in this study to highlight the variability existing in the spectral data set recorded for the different tests of the dengue infection, and to differentiate their spectroscopic signatures. PCA-FDA was employed to further discriminate spectra based on the levels of the markers of dengue infection, such as IgG, demonstrating the diagnostic potential of the technique.

Supervised learning methods such as discriminant analyses enable classification of unknown samples using prior knowledge of the category from training sets. To predict the qualitative affiliation of data points to a defined group (for instance level of the dengue antibody IgG), the coupling of PCA with Factorial Discriminant Analysis (PCA-FDA) exploits the calculated PC scores to better evaluate the discrimination rate achieved based on a data set [39].In the present study, leave one patient out strategy has been employed to discriminate between healthy and dengue blood plasma. The classification values obtained for each iteration have been gathered to deliver an overall confusion matrix representative of the data discrimination. For this type of strategy, the model is restricted to a specified number of iteration, dictated by the number of 
patients. In present study, there are 34 groups of spectra (17 healthy and 17 dengue). Thus, in each iteration, one is patient taken out as validation and the 33 remaining are used as calibration.

\begin{tabular}{|c|c|}
\hline Peak cm cm $^{-1}$ & Assignment \\
\hline 608 & Cholesterol \\
\hline 656 & Protiens(L-histidine) \\
\hline 688 & Glycine \\
\hline 708 & Tryptophan \\
\hline 802 & Uracil-based ring breathing mode \\
\hline 828 & Proline, hydroxyproline, tyrosine, $\mathrm{v}_{2} \mathrm{PO}_{2}^{-}$stretch of nucleic acids \\
\hline 849 & $\begin{array}{l}\text { Most probably due to single-bond stretching vibration for the amino acids and } \\
\text { valine and polysaccharides. Also assigned to proline, tyrosine, tryptophan-IgG }\end{array}$ \\
\hline 875 & $\begin{array}{l}\text { Antisymmetric stretch vibration of choline group } \mathrm{N}^{+}\left(\mathrm{CH}_{3}\right)_{3} \text {, characteristic of } \\
\text { phospholipids }\end{array}$ \\
\hline 880 & Tryptophan \\
\hline 1102 & O-P-O backbone stretch of DNA \\
\hline 1164 & C-H bending tyrosine \\
\hline 1239 & $\begin{array}{l}\text { Amide III and } \mathrm{CH}_{2} \text { wagging vibration from glycine backbone and proline side } \\
\text { chains }\end{array}$ \\
\hline 1297 & Lipids/Palmitic acid \\
\hline 1317 & Guanine (B, Z-marker) \\
\hline 1343 & $\mathrm{CH}_{3}, \mathrm{CH}_{2}$ wagging (collagen assignment) Glucose \\
\hline 1491 & $\begin{array}{l}\mathrm{C}-\mathrm{N} \text { stretching vibration coupled with the in-plane } \mathrm{C}-\mathrm{H} \text { bending in amino } \\
\text { radical cations }\end{array}$ \\
\hline 1507 & N-H bending \\
\hline 1540 & Amide carbonyl group vibration and aromatic hydrogens \\
\hline 1587 & Phenylalanine, Haemoglobin \\
\hline 1640 & Amide I band (protein band), $\mathrm{C}=\mathrm{O}$ coupled to $\delta \mathrm{NH}_{2}$ in plane \\
\hline 1680 & Amide I band (protein band) \\
\hline 1719 & Lipid \\
\hline 1736 & $\mathrm{C}=\mathrm{O}$ ester (lipids) \\
\hline
\end{tabular}

Table 2: Raman spectral peaks observed in the Raman spectra of healthy and dengue infected blood plasma samples.

\section{Results and Discussion}


Figure 1 plots the ELISA based serology results of all three biomarkers for all samples, demonstrating that there is no clear correlation between the relative levels of the different biomarkers across the sample set. As can also be seen from Table 1, there are many instances of high levels of one biomarker, and normal levels of one or both of the other two. The serology biomarkers are therefore not an unambiguous diagnostic test for the presence of dengue infection. Ns1 is an antigen while IgG and IgM are antibodies and their serological levels in plasma depend on the day of the infection, as antigen (Ns1) can be detected 4-5 days after infection and antibodies including IgM are detectable 3-5 days after infection, but are undetectable after 2-3 month, while $\mathrm{IgG}$ is detectable after one week to several months [40-42].

Figure 2 shows the mean Raman spectra of the healthy (control) and dengue infected blood plasma samples. The major differences in the Raman spectral features are labelled as vertical lines, and are assigned in Table 2. These spectral features can be considered as Raman spectral markers of biochemical changes associated with the dengue infection. Increases are seen in bands associated with the lipidic contents including $608 \mathrm{~cm}^{-1}, 875 \mathrm{~cm}^{-1}, 1297 \mathrm{~cm}^{-1}, 1719 \mathrm{~cm}^{-1}$ and $1736 \mathrm{~cm}^{-1}$. Notably, in the literature [38] these bands are associated specifically as cholesterol $\left(608 \mathrm{~cm}^{-1}\right)$, choline $\left(875 \mathrm{~cm}^{-1}\right)$, palmitic acid $\left(1297 \mathrm{~cm}^{-1}\right)$, glutamic acid $\left(1719 \mathrm{~cm}^{-1}\right)$ and ester groups $\left(1736 \mathrm{~cm}^{-1}\right)$, indicating an increase in lipidic content in dengue infected samples. The lipids provide sites for the virus to attach, and hence a high concentration of lipids in dengue infected blood plasma can be associated with the dengue infection [43]. Furthermore, the complement-fixing of antibodies IgM is greater for higher amount of cholesterol and the Raman feature at $608 \mathrm{~cm}^{-1}$ is consistent with a high concentration of cholesterol (lipids) in the Raman spectra of dengue samples, so this peak may be associated with the greater complement fixing of the $\operatorname{IgM}$ and hence development of the dengue disease [44].

Raman spectral features associated with proteins are also observed as prominent differentiating factors. The peak intensity of the feature at $1239 \mathrm{~cm}^{-1}$ (amide III $\left(\mathrm{CH}_{2}\right.$ wagging vibration)) is greater in the mean Raman spectra of dengue infected blood plasma. This has previously been associated with IgM [45]. There is also an increase in the intensity at $1409 \mathrm{~cm}^{-1}$ in dengue infected samples which can be associated with the $\operatorname{IgG}$ antibody $\left(\operatorname{IgG}\left(v_{s} \mathrm{COO}^{-1}\right)\right.$. The Raman spectral features at "608 (lipids), 688 (glycine), 708 (tryptophan), 1164 (C-H bending), 1297 (lipids), 1507 (protein A), 1640 and 1680 (amide-I) and $1719 \mathrm{~cm}-1$ (lipids) are observed with significantly higher 
intensities in dengue positive samples as compared to dengue negative samples/healthy ones and are also associated with proteins. Notably, although the samples were measured in their wet state, the characteristic, but weak, water feature at $\sim 1640 \mathrm{~cm}-1$ is not apparent in the spectrum of the control.

Other spectral features associated with proteins include those at 880(tryptophan), 656(L-histidine) and 1164(tyrosine), 1102(amide III of proteins), 1343( $\mathrm{CH}_{3}, \mathrm{CH}_{2}$ wagging), 1587(haemoglobin) and $1317 \mathrm{~cm}^{-1}$ (C-H deformation), and have higher intensity in dengue infected blood plasma samples than in healthy volunteers.

Notably, the Raman spectral features at 828 and $849 \mathrm{~cm}^{-1}$, associated with out of plane tyrosine ring breathing [46], have higher intensity in the mean Raman spectra of healthy than dengue infected samples. A small peak evident with higher intensity in dengue infected spectra at 1491 $\mathrm{cm}^{-1}$ is due the $\mathrm{C}-\mathrm{N}$ stretching vibration coupled with the in plane $\mathrm{C}-\mathrm{H}$ bending in amino radical cations. The peak at $1507 \mathrm{~cm}^{-1}$, again present with significantly higher intensity in the mean Raman spectra of dengue infected blood plasma, is due to N-H bending and has been reported for lysine present in protein A [47], which strongly binds IgG and makes variable binding with IgM [48]. The small peak at $1540 \mathrm{~cm}^{-1}$ which is significant in the dengue infected blood plasma is the Raman feature of the amide carbonyl group vibration and aromatic hydrogen and it has been reported that this peak is associated with the non structural protein (Ns1) [49]. The prominence of proteins in the Raman spectral data of dengue infected blood plasma can therefore be associated with the presence of dengue virus.

Figure 3 (a) presents the PCA scatter plot of Raman spectral data of blood plasma of healthy volunteers versus those of dengue patients, in which dengue infected spectra are clustered in the positive side of $\mathrm{x}$-axis, indicated by red dots and healthy blood plasma spectra are negative, shown by blue dots. A reasonably good separation of the datasets is indicated according to the $1^{\text {st }}$ principle component (PC1 -77.42\% of variance). The PCA loading associated with PC1 is shown in Figure 3 (b), in which the positive loadings can be associated with the Raman spectral data clustered in the positive axis of the PC-1 and the negative loadings can be associated with the Raman spectral data clustered in the negative side of the $\mathrm{x}$-axis [50].The major features of the PCA loading plot are almost the same as the differences observed in the Raman mean spectra of healthy verses dengue (Figure 1). The PCA loading plot shows differences at 608, 1297, 1409, 1719 and 1736 
$\mathrm{cm}^{-1}$, also found in the mean Raman spectra of dengue infected blood plasma. These are the Raman features for lipids which can be associated with the anti-bodies ( $\operatorname{IgM}$ and $\operatorname{IgG}$ ) produced against infection. Similarly, the differences at $880,1239,1491,1507,1540,1640$, and $1680 \mathrm{~cm}^{-1}$ in the PCA loadings plot on the positive $\mathrm{x}$-axis of PC-1 are the Raman signals for proteins. These features may be considered as identification markers of dengue virus infection and have been reported at $1540 \mathrm{~cm}^{-1}$ for Ns1 in earlier research [49]. So, it can be extrapolated that other Raman spectral features like $880,1491,1507,1640$, and $1680 \mathrm{~cm}^{-1}$ may also be indication of dengue virus infection.

As shown in Figure 1, there is little or no correlation between the levels of the three biomarkers, as determined by ELISA. Similarly, the distribution of the Raman spectra in the PCA of the dengue samples versus control shows no correlation with biomarker levels, as indicated in the Supplemental Material, Figures S1-3. Notably, samples with low levels of biomarkers are often well discriminated from the controls, indicating that Raman Spectroscopic analysis provides a more holistic analysis of the biochemical content of the blood sample.

In order to quantify the diagnostic potential of the spectroscopic technique, a further classification of the control and dengue groups using PCA-FDA was carried out. The results are shown in Table 3, which indicates that, based on the spectroscopic analysis of the current, albeit limited, cohort of samples, Principal Components Analysis (PCA) coupled with Factorial Discriminant Analysis, values of $97.95 \%$ sensitivity and $95.40 \%$ specificity for identification of dengue infection can be achieved., which is comparable with the sensitivity of $98 \%$ and specificity of $100 \%$ of dengue kit, as quoted on the dengue-kit used for the current study (Vircell Cat No. G1018, M1018).

Notably, however, each test needs to be performed separately and independently for each sample, and is thus more demanding on consumables and therefore cost, whereas the Raman assay is label free, and simply requires the plasma from the blood of the dengue patients.

\begin{tabular}{|l|l|l|l|l|}
\hline & Dengue & Normal & Total/group & Specificity \\
\hline Dengue & 383 & 16 & 391 & $95.40 \%$ \\
\hline
\end{tabular}




\begin{tabular}{|l|l|l|l|l|}
\hline Normal & 8 & 332 & 348 & Sensitivity \\
\hline & & Total spectra & 739 & $97.95 \%$ \\
\hline
\end{tabular}

Table 3: Results of the PCA-FDA for the Raman spectral data of dengue positive and normal blood plasma samples.

Performed individually, any one of the ELISA based biomarker tests can indicate low levels of infection, whereas another of the tests indicates high. For the example of IgM screening of the patient cohort represented in Table 1, 7 patients are diagnosed as "Low", for which the other two tests yield a value of "high" or "medium", indicating a sensitivity of this test alone of as low as $63 \%$. As a further demonstration of the potential of a Raman based assay, the control samples were compared with those samples which registered low for only one biomarker, but not the other two, by employing PCA and PCA-FDA. The samples selected in this way thus include Low Ns1 (D5, D6), Low IgM (D2, D3, D10) and Low IgG (D7, D11, D16) (Table 1).

Figure 4 shows the PCA scatter plot of the Raman spectral data of control, versus the reduced dataset of blood plasma samples from dengue patients. There is reasonably good separation of the controls versus low levels of these three biomarkers of dengue by PC-1 (80.36\%). Notably, as all the three biomarkers are uncorrelated with each other as the samples which have low levels of one biomarker may have medium or high levels of other biomarker, they do not appear here as separate clusters in the PCA scatter plot. PCA-FDA results, for the comparison of Normal samples with the dataset of all lows mixed (Ns1, IgM and IgG) are shown in Table 4, which indicates that the presence of dengue infection in blood samples can be identified with a sensitivity of $97.38 \%$ and a specificity of $86.18 \%$, in samples for which one of the biomarkers scored low.

\begin{tabular}{|l|l|l|l|l|}
\hline & $\begin{array}{l}\text { Dengue } \\
\text { (All lows })\end{array}$ & Normal & Total/group & Specificity \\
\hline
\end{tabular}




\begin{tabular}{|l|l|l|l|l|}
\hline $\begin{array}{l}\text { Dengue } \\
\text { (All lows) }\end{array}$ & 186 & 5 & 191 & $86.18 \%$ \\
\hline Normal & 47 & 293 & 340 & Sensitivity \\
\hline & & Total spectra & 531 & $97.38 \%$ \\
\hline
\end{tabular}

Table 4: Results of the PCA-FDA for the Raman spectral data of Normal versus all lows mixed (Ns1,IgM and IgG) samples.

\section{Conclusions:}

The potential of Raman spectroscopy for the diagnosis and characterisation of healthy and dengue infected human blood plasma samples has been demonstrated. The spectral analysis indicates that distinct Raman spectral features are observed in the dengue positive samples, potentially associated with the development of the disease, as compared to the healthy samples. These Raman spectral features hence can be considered as markers of the dengue development and can be helpful for the diagnosis of the disease. Multivariate analysis with PCA and PCA-FDA enables a more precise identification of these spectral markers, and also allows a quantification of the diagnostic potential. Notably, as the Raman analysis is not based specifically on an individual biomarker, it provides a more holistic view of the biochemical content of the sample, and abnormalities associated with the disease. Critically, it identifies the presence of the disease in samples for which at least one of the biomarker tests indicated low levels, and thus can be considered an alternative for improved, unambiguous screening.

\section{Conflict of Interest}

The authors declare that there is no conflict of interest for this research work.

\section{References}

[1] S. Bhatt, P.W. Gething, O.J. Brady, J.P. Messina, A.W. Farlow, C.L. Moyes, J.M. Drake, J.S. Brownstein, A.G. Hoen, O. Sankoh, The global distribution and burden of dengue, Nature, 496 (2013) 504-507.

[2] D. Subedi, A.W. Taylor-Robinson, Laboratory diagnosis of dengue infection: Current techniques and future strategies, Open Journal of Clinical Diagnostics, 2014 (2014). 
[3] C.C. Carlos, K. Oishi, M.T. Cinco, C.A. Mapua, S. Inoue, D.J.M. Cruz, M.A.M. Pancho, C.Z. Tanig, R.R. Matias, K. Morita, Comparison of clinical features and hematologic abnormalities between dengue fever and dengue hemorrhagic fever among children in the Philippines, The American journal of tropical medicine and hygiene, 73 (2005) 435-440.

[4] M.G. Guzmán, G. Kouri, J. Bravo, L. Valdes, V. Susana, S.B. Halstead, Effect of age on outcome of secondary dengue 2 infections, International journal of infectious diseases, 6 (2002) 118-124.

[5] L. Kittigul, P. Pitakarnjanakul, D. Sujirarat, K. Siripanichgon, The differences of clinical manifestations and laboratory findings in children and adults with dengue virus infection, Journal of Clinical Virology, 39 (2007) 76-81.

[6] S. Yacoub, P.K. Lam, L.H.M. Vu, T.L. Le, N.T. Ha, T.T. Toan, N.T. Van, N.T.H. Quyen, H.T. Le Duyen, N. Van Kinh, Association of microvascular function and endothelial biomarkers with clinical outcome in dengue: an observational study, The Journal of infectious diseases, 214 (2016) 697-706.

[7] A. Maeda, J. Maeda, Review of diagnostic plaque reduction neutralization tests for flavivirus infection, The Veterinary Journal, 195 (2013) 33-40.

[8] S. Alcon, A. Talarmin, M. Debruyne, A. Falconar, V. Deubel, M. Flamand, Enzyme-linked immunosorbent assay specific to Dengue virus type 1 nonstructural protein NS1 reveals circulation of the antigen in the blood during the acute phase of disease in patients experiencing primary or secondary infections, Journal of clinical microbiology, 40 (2002) 376-381.

[9] S. Datta, C. Wattal, Dengue NS1 antigen detection: A useful tool in early diagnosis of dengue virus infection, Indian journal of medical microbiology, 28 (2010) 107.

[10] F.M. Kassim, M.N. Izati, T. TgRogayah, Y.M. Apandi, Z. Saat, Use of dengue NS1 antigen for early diagnosis of dengue virus infection, Southeast Asian Journal of Tropical Medicine and Public Health, 42 (2011) 562.

[11] S. Zainah, A.A. Wahab, M. Mariam, M. Fauziah, A. Khairul, I. Roslina, A. Sairulakhma, S. Kadimon, M.M. Jais, K. Chua, Performance of a commercial rapid dengue NS1 antigen immunochromatography test with reference to dengue NS1 antigen-capture ELISA, Journal of virological methods, 155 (2009) 157-160. [12] T. Jaenisch, D.T.H. Tam, N.T.T. Kieu, T. Ngoc, N.T. Nam, N. Van Kinh, S. Yacoub, N. Chanpheaktra, V. Kumar, L.L.C. See, Clinical evaluation of dengue and identification of risk factors for severe disease: protocol for a multicentre study in 8 countries, BMC infectious diseases, 16 (2016) 120.

[13] E.F.B. SHAIR, A NOVEL APPROACH TO PREDICT RISK IN DENGUE HEMORRHAGIC FEVER (DHF) USING NEURAL NETWORK, in, Universiti Teknologi Malaysia, 2009.

[14] W.H. Organization, Dengue guidelines for diagnosis, treatment, prevention and control: new edition, (2009).

[15] W.H. Organization, S.P.f. Research, T.i.T. Diseases, W.H.O.D.o.C.o.N.T. Diseases, W.H.O. Epidemic, P. Alert, Dengue: guidelines for diagnosis, treatment, prevention and control, World Health Organization, 2009.

[16] V. Wiwanitkit, Dengue fever: diagnosis and treatment, Expert review of anti-infective therapy, 8 (2010) 841-845.

[17] P. Crow, J. Uff, J. Farmer, M. Wright, N. Stone, The use of Raman spectroscopy to identify and characterize transitional cell carcinoma in vitro, BJU international, 93 (2004) 1232-1236.

[18] C.A. Owen, I. Notingher, R. Hill, M. Stevens, L.L. Hench, Progress in Raman spectroscopy in the fields of tissue engineering, diagnostics and toxicological testing, Journal of Materials Science: Materials in Medicine, 17 (2006) 1019-1023.

[19] F.M. Lyng, E.Ó. Faoláin, J. Conroy, A. Meade, P. Knief, B. Duffy, M. Hunter, J. Byrne, P. Kelehan, H. Byrne, Vibrational spectroscopy for cervical cancer pathology, from biochemical analysis to diagnostic tool, Experimental and molecular pathology, 82 (2007) 121-129. 
[20] P. Knief, C. Clarke, E. Herzog, M. Davoren, F.M. Lyng, A.D. Meade, H.J. Byrne, Raman spectroscopy-a potential platform for the rapid measurement of carbon nanotube-induced cytotoxicity, Analyst, 134 (2009) 1182-1191.

[21] J. Chan, S. Fore, S. Wachsmann-Hogiu, T. Huser, Raman spectroscopy and microscopy of individual cells and cellular components, Laser \& Photonics Reviews, 2 (2008) 325-349.

[22] Z. Farhane, F. Bonnier, A. Casey, H.J. Byrne, Raman micro spectroscopy for in vitro drug screening: subcellular localisation and interactions of doxorubicin, Analyst, 140 (2015) 4212-4223.

[23] T. Huser, C.A. Orme, C.W. Hollars, M.H. Corzett, R. Balhorn, Raman spectroscopy of DNA packaging in individual human sperm cells distinguishes normal from abnormal cells, Journal of biophotonics, 2 (2009) 322.

[24] A.D. Meade, C. Clarke, F. Draux, G.D. Sockalingum, M. Manfait, F.M. Lyng, H.J. Byrne, Studies of chemical fixation effects in human cell lines using Raman microspectroscopy, Analytical and bioanalytical chemistry, 396 (2010) 1781-1791.

[25] A.D. Meade, F.M. Lyng, P. Knief, H.J. Byrne, Growth substrate induced functional changes elucidated by FTIR and Raman spectroscopy in in-vitro cultured human keratinocytes, Analytical and bioanalytical chemistry, 387 (2007) 1717-1728.

[26] H. Nawaz, F. Bonnier, P. Knief, O. Howe, F.M. Lyng, A.D. Meade, H.J. Byrne, Evaluation of the potential of Raman microspectroscopy for prediction of chemotherapeutic response to cisplatin in lung adenocarcinoma, Analyst, 135 (2010) 3070-3076.

[27] H. Nawaz, F. Bonnier, A.D. Meade, F.M. Lyng, H.J. Byrne, Comparison of subcellular responses for the evaluation and prediction of the chemotherapeutic response to cisplatin in lung adenocarcinoma using Raman spectroscopy, Analyst, 136 (2011) 2450-2463.

[28] H. Nawaz, A. Garcia, A.D. Meade, F.M. Lyng, H.J. Byrne, Raman micro spectroscopy study of the interaction of vincristine with A549 cells supported by expression analysis of bcl-2 protein, Analyst, 138 (2013) 6177-6184.

[29] M. Saleem, M. Bilal, S. Anwar, A. Rehman, M. Ahmed, Optical diagnosis of dengue virus infection in human blood serum using Raman spectroscopy, Laser Physics Letters, 10 (2013) 035602.

[30] K.M. Ostrowska, A. Garcia, A.D. Meade, A. Malkin, I. Okewumi, J.J. O'Leary, C. Martin, H.J. Byrne, F.M. Lyng, Correlation of p16 INK4A expression and HPV copy number with cellular FTIR spectroscopic signatures of cervical cancer cells, Analyst, 136 (2011) 1365-1373.

[31] K.M. Ostrowska, A. Malkin, A. Meade, J. O'Leary, C. Martin, C. Spillane, H.J. Byrne, F.M. Lyng, Investigation of the influence of high-risk human papillomavirus on the biochemical composition of cervical cancer cells using vibrational spectroscopy, Analyst, 135 (2010) 3087-3093.

[32] H. Nawaz, N. Rashid, M. Saleem, M. Asif Hanif, M. Irfan Majeed, I. Amin, M. Iqbal, M. Rahman, O. Ibrahim, S. Baig, Prediction of viral loads for diagnosis of Hepatitis $\mathrm{C}$ infection in human plasma samples using Raman spectroscopy coupled with partial least squares regression analysis, Journal of Raman Spectroscopy, 48 (2017) 697-704.

[33] S. Khan, R. Ullah, A. Khan, N. Wahab, M. Bilal, M. Ahmed, Analysis of dengue infection based on Raman spectroscopy and support vector machine (SVM), Biomedical optics express, 7 (2016) 2249-2256. [34] M. Bilal, M. Saleem, M. Bilal, T. Ijaz, S. Khan, R. Ullah, A. Raza, M. Khurram, W. Akram, M. Ahmed, Raman spectroscopy-based screening of IgM positive and negative sera for dengue virus infection, Laser Physics, 26 (2016) 115602.

[35] J. De Gelder, K. De Gussem, P. Vandenabeele, L. Moens, Reference database of Raman spectra of biological molecules, Journal of Raman Spectroscopy, 38 (2007) 1133-1147.

[36] I. Notingher, Raman spectroscopy cell-based biosensors, Sensors, 7 (2007) 1343-1358.

[37] P. Jess, V. Garcés-Chávez, D. Smith, M. Mazilu, L. Paterson, A. Riches, C. Herrington, W. Sibbett, K. Dholakia, Dual beam fibre trap for Raman microspectroscopy of single cells, Optics Express, 14 (2006) 5779-5791. 
[38] C. Krafft, L. Neudert, T. Simat, R. Salzer, Near infrared Raman spectra of human brain lipids, Spectrochimica Acta Part A: Molecular and Biomolecular Spectroscopy, 61 (2005) 1529-1535.

[39] D. Bertrand, P. Courcoux, J.C. Autran, R. Meritan, P. Robert, Stepwise canonical discriminant analysis of continuous digitalized signals: Application to chromatograms of wheat proteins, Journal of Chemometrics, 4 (1990) 413-427.

[40] B. Innis, A. Nisalak, S. Nimmannitya, S. Kusalerdchariya, V. Chongswasdi, S. Suntayakorn, P. Puttisri, C. Hoke, An enzyme-linked immunosorbent assay to characterize dengue infections where dengue and Japanese encephalitis co-circulate, The American journal of tropical medicine and hygiene, 40 (1989) 418427.

[41] D. PAHO, dengue hemorrhagic fever in the Americas: Guidelines for prevention and control, Scientific Publication No. 548, Pan American Health Organization, (1994).

[42] D.H. Fever, Diagnosis, Treatment, Prevention and Control. Geneva: World Health Organization (WHO) and Special Programme for Research and Training in Tropical Diseases (TDR), 3 (2009).

[43] J. McLauchlan, Lipid droplets and hepatitis C virus infection, Biochimica et Biophysica Acta (BBA)Molecular and Cell Biology of Lipids, 1791 (2009) 552-559.

[44] G.M. Swartz, M.K. Gentry, L.M. Amende, E.J. Blanchette-Mackie, C.R. Alving, Antibodies to cholesterol, Proceedings of the National Academy of Sciences, 85 (1988) 1902-1906.

[45] P.C. Painter, J. Koenig, Raman spectroscopic study of the structure of antibodies, Biopolymers, 14 (1975) 457-468.

[46] Z. Movasaghi, S. Rehman, I.U. Rehman, Raman spectroscopy of biological tissues, Applied Spectroscopy Reviews, 42 (2007) 493-541.

[47] Z.A. Combs, S. Chang, T. Clark, S. Singamaneni, K.D. Anderson, V.V. Tsukruk, Label-free raman mapping of surface distribution of protein A and IgG biomolecules, Langmuir, 27 (2011) 3198-3205.

[48] E.H. Sasso, G.J. Silverman, M. Mannik, Human IgA and IgG F ( $\left.a b^{\prime}\right) 2$ that bind to staphylococcal protein A belong to the VHIII subgroup, The Journal of Immunology, 147 (1991) 1877-1883.

[49] A. Radzol, K.Y. Lee, W. Mansor, Nonstructural protein 1 characteristic peak from NS1-saliva mixture with Surface-Enhanced Raman spectroscopy, in: Engineering in Medicine and Biology Society (EMBC), 2013 35th Annual International Conference of the IEEE, IEEE, 2013, pp. 2396-2399.

[50] F. Bonnier, H. Byrne, Understanding the molecular information contained in principal component analysis of vibrational spectra of biological systems, Analyst, 137 (2012) 322-332. 